\title{
Power Flows and Efficiency of Output Compound e-CVT
}

\author{
Francesco Bottiglione and Giacomo Mantriota \\ Dipartimento di Meccanica, Matematica e Management, Politecnico di Bari, Viale Japigia 182, 70125 Bari, Italy \\ Correspondence should be addressed to Giacomo Mantriota; giacomo.mantriota@poliba.it
}

Received 9 April 2015; Revised 15 July 2015; Accepted 20 August 2015

Academic Editor: Shankar Subramanian

Copyright (c) 2015 F. Bottiglione and G. Mantriota. This is an open access article distributed under the Creative Commons Attribution License, which permits unrestricted use, distribution, and reproduction in any medium, provided the original work is properly cited.

\begin{abstract}
Hybridization is the most promising vehicular technology to get significant improvements of the vehicle efficiency and performance in the short-term. Mechanical transmissions for hybrid vehicles are very often multiple modes transmission, which permit improving the performance in different working conditions. In this context, optimal design and control of these transmissions are a key point to improve the performance of the vehicles, and mathematical models which supports the design can play an important role in this field. In this work, an approach for evaluating the performance of Output Compound Split e-CVT (electric Continuously Variable Transmission) in steady-state is proposed. This approach, in addition to a kinematic analysis of the device, leads to the calculation of the internal power circulation modes and the efficiency of the device in different working conditions.
\end{abstract}

\section{Introduction}

Hybrid Electric Vehicles (HEVs) represent one of the most promising fuel-saving technologies in the short-term for improving fuel economy of ground vehicles [1]. Due to their significant potential in reducing fuel consumption and emissions, many car companies now actively develop HEVs. Hybrid vehicles work in several operating conditions: full electric condition, charging of battery condition, stationary condition, recovering of the energy from brakes condition. The operating mode control strategy is of great importance to take the best from the HEV [2-6].

Because of the multiple power sources of the HEVs, transmissions with multiple ports are often employed as, for example, the Power Split Continuously Variable Transmissions (PS-CVT). Among these, the Input Split type has good efficiency in the overall shifting range. For this reason, it is the most suitable power split system for single mode hybrid powertrains. However, single mode powertrains of Input Split type show low efficiency at high vehicle speeds [79]. In dual mode powertrains, the power split type is selected among the two, engaging and disengaging a clutch [10-15]. The use of two modes overcomes the problems of the single mode powertrain, providing better vehicle performance in terms of fuel economy, acceleration, and motor size. In fact, multiple-port multiple-mode transmissions permit reducing the power through the electrical path, of which the typical efficiency is $75 \%$, and increasing the power through the more efficient mechanical path (95\%), thus increasing the efficiency of the overall vehicle powertrain $[14,15]$.

Besides the input power split CVT, there are several more complex solutions, based on the use of at least two epicyclical gear trains and one or more locking systems. These solutions can be classified as compound type power split, and examples are given by the devices developed by Allison, Timken, Renault, and Toyota and by the GM/Daimler/BMW joint project called Global Hybrid Cooperation [16]. A proper modeling and simulation tool is very important in the early design and analysis stage. This is even more critical for the Compound Split Power Split Electric CVTs (eCVTs) since there could be numerous possible configurations/components and various control strategies [17-21].

PS-CVTs have been studied in many works, with focus on the efficiency and the development of original types [22-26]. It has been demonstrated that a fuel economy improvement can be obtained through a PS-CVT in vehicles with internal combustion engine [27]. Depending on the location of the PG drive they can be distinguished in Output Split (or input coupled) and Input Split (or output coupled) devices. Furthermore, depending on the ratio range of the PS-CVT 


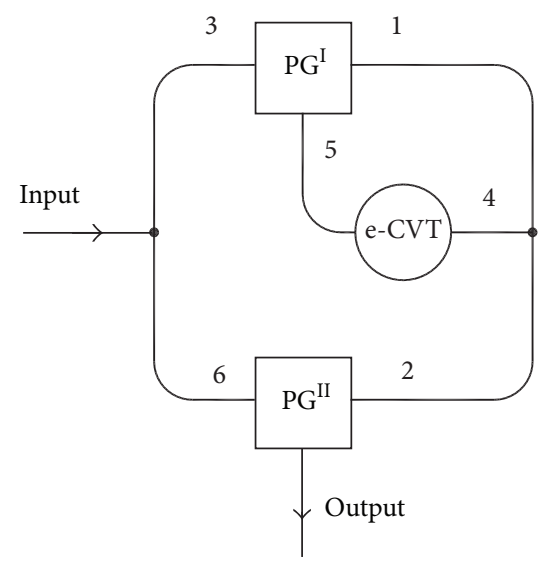

(a)

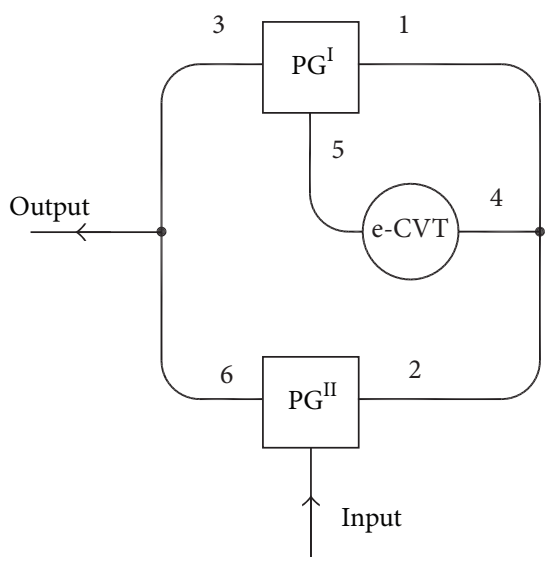

(b)

Figure 1: Schematic picture of the Output Compound Split Transmission (a) and Input Compound Split Transmission (b).

and of the Continuously Variable Transmission, different internal power flows may be established, which affects the ratio between the power through the CVT branch and the power transmitted by the device.

In hybrid vehicles, during the recovery of energy in braking, the transmission works in reverse mode and it has been shown that the efficiency in reverse mode is sometimes critically lower than the efficiency in direct mode [22].

Compound Split Transmission uses two motor/generators as variator (eCVT), as well as one or several planetary gear sets as power split device. These architectures can work in two different modes by searching for the best global efficiency in the different operating conditions.

A simple approach would be useful to determine the performance of compound Electric CVT, in order to optimize the control strategy and then the performance of hybrid vehicles [26].

Following this approach, it would be possible to predict the power flows of the transmission and its efficiency. Optimal control strategies of hybrid vehicles are very important. Several studies were carried out in considering the design, efficiency, and mathematical models for the control strategy. The focus of these surveys is the fuel consumption and the performance of the hybrid vehicle.

In this work, a method to evaluate the power flows and the efficiency of Output Compound Split Transmission is proposed (Figure 1(a)). The Output Compound architecture is made of a planetary gear $\left(\mathrm{PG}^{\mathrm{I}}\right)$, input shaft, and an e-CVT connected by a second planetary gear $\left(\mathrm{PG}^{\mathrm{II}}\right)$ (Figure $1(\mathrm{a})$ ). For the first time the Output Compound architecture is here studied by separating the system into two subsystems. Following this approach, the Output Compound is made of two nested shunted CVT systems of Input or Output Split type, to be analyzed through a previously developed approach. It follows that, after having imposed the requested ratio ranges, the power circulation modes are easily found and also the efficiency can be easily calculated. It can be observed that the reverse operation of the Output Compound Split system is closely related to the Input Compound Split (Figure 1(b)) that one of the authors has studied in a previous work. Such operational conditions are typical of the regenerative braking where the power flows backwards with respect to the normal operation mode of the transmission. As it is shown in a previous work, the efficiency of the Power Split Transmission is very different in reverse operation in comparison to direct operation. For this reason, in this work the efficiency of the Output Split transmission will be investigated and a comparison between the direct and the reverse operations will be achieved.

\section{Kinematics of Output Compound Split Transmission}

A compound power split is made of a continuously variable drive, which can be of mechanical type (CVT) or with two motors/generators working as electrical CVT (e-CVT), as well as one or several planetary gear sets. They can be classified into three different types: Input Split, Output Split, and Compound Split. The Input and Output Split configurations have only one planetary gear, operating as a power split device. In the Compound Split Transmission the planetary gears are two or more. Hereafter, we will consider Compound Split Transmissions with two planetary gear sets ( $\mathrm{PG}^{\mathrm{I}}$ and $\left.P G^{I I}\right)$. Many different combinations can be achieved with different arrangements of the connections between all the elements of the transmission. Among all possibilities, the two most diffused are shown in Figure 1: the Output Compound Split Transmission (Figure 1(a)) and the Input Compound Split Transmission (Figure 1(b)). The Input Compound Split Transmission was studied in a previous work of one of the authors (G. Mantriota). In the present work, the Output Compound Split Transmission is investigated. These configurations can be considered one the reverse mode operations of the other. Since the object of our study is a Continuously Variable Unit (CVU) made with a Compound Split Transmission, hereafter, we will refer to this with the acronym CVU. 
Derivation of the main kinematic relations for the Output Compound Split Transmission (Figure 1(a)) follows. By defining $k_{1}$ and $k_{2}$ the gear ratios of the planetary gears PG1 and PG2, respectively, the following algebraic equations hold:

$$
\begin{aligned}
& \omega_{5}+k_{1} \omega_{1}-\left(1+k_{1}\right) \omega_{3}=0, \\
& \omega_{o}+k_{2} \omega_{6}-\left(1+k_{2}\right) \omega_{2}=0,
\end{aligned}
$$

where $\omega_{j}$ is the angular speed of the $j$ th path.

The speed ratio of the compound transmission $\tau_{\mathrm{CVU}}$ and the speed ratio of the eCVT are

$$
\begin{gathered}
\tau_{\mathrm{CVU}}=\frac{\omega_{o}}{\omega_{i}} ; \\
\tau_{\mathrm{ECVT}}=\frac{\omega_{5}}{\omega_{4}},
\end{gathered}
$$

where $\omega_{i}, \omega_{o}$ are the angular velocities of the input and the output shafts of the CVU. From (1)-(3) and observing that $\omega_{3}=\omega_{i}=\omega_{6}$ and $\omega_{1}=\omega_{2}=\omega_{4}$ (Figure 1(a)), the speed ratio of the CVU is obtained:

$$
\tau_{\mathrm{CVU}}=\frac{1+k_{1}+k_{2}\left(1-\tau_{\mathrm{ECVT}}\right)}{\tau_{\mathrm{ECVT}}+k_{1}} .
$$

Making the derivative of (4) with respect to $\tau_{\mathrm{ECVT}}$,

$$
\frac{d \tau_{\mathrm{CVU}}}{d \tau_{\mathrm{ECVT}}}=-\frac{k_{1}+k_{2}+k_{2} k_{1}}{\left(\tau_{\mathrm{ECVT}}+k_{1}\right)^{2}}
$$

then the CVU speed ratio is always a monotonic function since the sign of the derivate (5) does not change if $\tau_{\mathrm{ECVT}}$ changes.

If one desires specific minimum $\left(\tau_{\mathrm{CVU}}\right)$ and maximum $\left(\tau_{\mathrm{CVUM}}\right)$ values of the global speed ratio with given $\tau_{\mathrm{ECVT} m}$ and $\tau_{\text {ECVT }}$, it is possible to calculate $k_{1}$ and $k_{2}$ as the unique solution of a system of two equations with two unknowns. In fact, if $\tau_{\mathrm{CVU}}$ is supposed to be a monotonic increasing function of $\tau_{\mathrm{ECVT}}$, then the following system of equations can be written:

$$
\begin{aligned}
& k_{1}\left(\tau_{\mathrm{CVU} M}-1\right)+k_{2}\left(\tau_{\mathrm{ECVT} M}-1\right)+\tau_{\mathrm{CVU} M} \tau_{\mathrm{ECVT} M} \\
& \quad-1=0, \\
& k_{1}\left(\tau_{\mathrm{CVU}_{m}}-1\right)+k_{2}\left(\tau_{\mathrm{ECVT}_{m}}-1\right)+\tau_{\mathrm{CVU}_{m}} \tau_{\mathrm{ECVT} m} \\
& \quad-1=0 .
\end{aligned}
$$

From it, it follows that

$$
\begin{aligned}
& k_{1}=\frac{\left(\tau_{\mathrm{CVU} M} \tau_{\mathrm{ECVT} M}-1\right)\left(1-\tau_{\mathrm{ECVT} m}\right)-\left(\tau_{\mathrm{CVU} m} \tau_{\mathrm{ECVT} m}-1\right)\left(1-\tau_{\mathrm{ECVT} M}\right)}{\left(\tau_{\mathrm{CVU} M}-1\right)\left(\tau_{\mathrm{ECVT} m}-1\right)-\left(\tau_{\mathrm{CVU} m}-1\right)\left(\tau_{\mathrm{ECVT} M}-1\right)}, \\
& k_{2}=\frac{\left(\tau_{\mathrm{CVU} M} \tau_{\mathrm{ECVT} M}-1\right)\left(1-\tau_{\mathrm{CVU}}\right)-\left(\tau_{\mathrm{CVU}_{m}} \tau_{\mathrm{ECVT} m}-1\right)\left(1-\tau_{\mathrm{CVU} M}\right)}{\left(\tau_{\mathrm{CVU} m}-1\right)\left(\tau_{\mathrm{ECVT} M}-1\right)-\left(\tau_{\mathrm{CVU} M}-1\right)\left(\tau_{\mathrm{ECVT} m}-1\right)} .
\end{aligned}
$$

Then, the values of the parameters $k_{1}$ and $k_{2}$ of the planetary gears depend only on the limits imposed of $\tau_{\mathrm{ECVT}}$ and $\tau_{\mathrm{CVU}}$. $k_{1}$ and $k_{2}$ can also be obtained in case that $\tau_{\mathrm{CVU}}$ is supposed to be a monotonic decreasing function of $\tau_{\mathrm{ECVT}}$ :

$$
\begin{aligned}
& k_{1}=\frac{\left(\tau_{\mathrm{CVU} M} \tau_{\mathrm{ECVT} m}-1\right)\left(1-\tau_{\mathrm{ECVT} M}\right)-\left(\tau_{\mathrm{CVU} m} \tau_{\mathrm{ECVT} M}-1\right)\left(1-\tau_{\mathrm{ECVT} m}\right)}{\left(\tau_{\mathrm{CVU} M}-1\right)\left(\tau_{\mathrm{ECVT} M}-1\right)-\left(\tau_{\mathrm{CVU} m}-1\right)\left(\tau_{\mathrm{ECVT} m}-1\right)}, \\
& k_{2}=\frac{\left(\tau_{\mathrm{CVU}_{M}} \tau_{\mathrm{ECVT}_{m}}-1\right)\left(1-\tau_{\mathrm{CVU}_{m}}\right)-\left(\tau_{\mathrm{CVU}_{m}} \tau_{\mathrm{ECVT} M}-1\right)\left(1-\tau_{\mathrm{CVU} M}\right)}{\left(\tau_{\mathrm{CVU}_{m}}-1\right)\left(\tau_{\mathrm{ECVT}}-1\right)-\left(\tau_{\mathrm{CVU} M}-1\right)\left(\tau_{\mathrm{ECVT} M}-1\right)} .
\end{aligned}
$$

In conclusion, the values of $\tau_{\mathrm{CVU}}$ corresponding to working conditions with zero power through the eCVT (mechanical points) are obtained from (4) with $\omega_{5}=0$ (corresponding to $\tau_{\mathrm{ECVT}}=\infty$ ) or $\omega_{4}=0$ (which corresponds to $\tau_{\mathrm{ECVT}}=0$ ):

$$
\begin{aligned}
& \tau_{\mathrm{CVU}}^{\mathrm{I}}=-k_{2} ; \\
& \tau_{\mathrm{CVU}}^{\mathrm{II}}=\frac{1+k_{1}+k_{2}}{k_{1}} .
\end{aligned}
$$

\section{Power Flow and Efficiency of Output Compound e-CVT}

In order to investigate the power flows and the efficiency of the Output Compound Split (Figure 1(a)), the overall system is divided into a subcomponent (Equivalent e-CVT) and the second planetary gear $\mathrm{PG}^{\mathrm{II}}$ (Figure $2(\mathrm{a})$ ). The Equivalent eCVT is made of the e-CVT and the $\mathrm{PG}^{\mathrm{I}}$ (Figure 2(b)). 


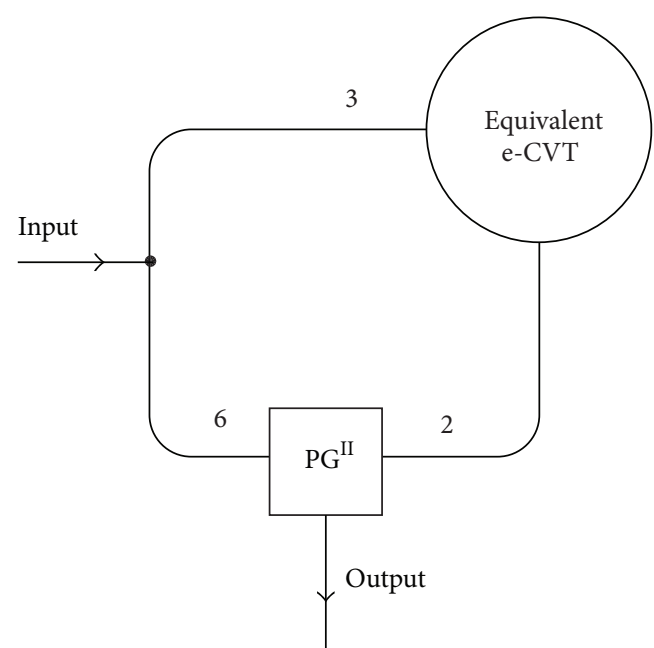

(a)

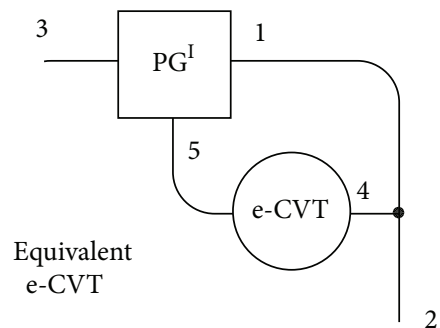

(b)

Figure 2: (a) Schematic picture of the Output Compound with the "Equivalent e-CVT." (b) Equivalent e-CVT.

The scheme of Figure 2(a) is also an Output Split (OS), where the Continuously Variable Transmission is replaced with the Equivalent e-CVT.

For this architecture, we define

$$
\tau_{\mathrm{ECVT}, \mathrm{eq}}=\frac{\omega_{3}}{\omega_{2}} .
$$

From (2)-(3) and (10) and considering $\omega_{3}=\omega_{6}=\omega_{i}$ (Figure 2(a)), it follows that

$$
\tau_{\mathrm{ECVT}, \mathrm{eq}}=\frac{\tau_{\mathrm{ECVT}}+k_{1}}{1+k_{1}} .
$$

The transmission ratio, in (11), depends only on the values of the parameter $k_{1}$ of the $\mathrm{PG}^{\mathrm{I}}$, and it is a monotonic function. From (2), (3), and (11) the CVU speed ratio is obtained as a function of the Equivalent e-CVT speed ratio:

$$
\tau_{\mathrm{CVU}}=\frac{1+k_{2}\left(1-\tau_{\mathrm{ECVT}, \mathrm{eq}}\right)}{\tau_{\mathrm{ECVT}, \mathrm{eq}}} .
$$

It has been demonstrated that, in the case of an Output Split (OS) (Figure 2(a)), a Type I power flow is obtained if $\left|\tau_{\mathrm{CVU}}\right|=\left|\omega_{o} / \omega_{i}\right|$ is a monotonic increasing function of $\left|\tau_{\mathrm{ECVT}}\right|=\left|\omega_{5} / \omega_{4}\right|$, whereas Type II power flow is obtained for a monotonic decreasing function.

In the case of Input Split, the power flows are the opposite with respect to the Output Split. This means that a Type I power flow can be obtained by imposing a monotonic decreasing trend of $\left|\tau_{\mathrm{CVU}}\right|=\left|\omega_{o} / \omega_{i}\right|$ in function of $\left|\tau_{\mathrm{ECVT}}\right|=$ $\left|\omega_{5} / \omega_{4}\right|$, whereas a Type II power flow is achieved if the function has a monotonic increasing trend.

Now, the power flows in the CVU can be analyzed as they are obtained as combinations of the power flows in the Output Split and Equivalent e-CVT. The power circulation in the compound depends only on whether the speed ratios of the CVU and Equivalent e-CVT are increasing or decreasing functions of Equivalent e-CVT and e-CVT, respectively.
If a monotonic increase of $\tau_{\mathrm{CVU}}$ as a function of $\tau_{\mathrm{ECVT}, \mathrm{eq}}$ is considered, a Type I power flow (Figure 3(b)) is obtained, that is, the power through the $\mathrm{PG}^{\mathrm{II}}$ is greater than the input power. In this condition, the Equivalent e-CVT is in an Output Split. If $\tau_{\mathrm{ECVT} \text {,eq }}$ is an increasing function of $\tau_{\mathrm{ECVT}}$, then a Type I power flow is obtained also in the Equivalent eCVT (Figure 3(c)). Therefore, this kind of power circulation, shown in Figure 3(a), is named Type I.I power flow. In the Type I.I power flow, also the overall speed ratio $\tau_{\mathrm{CVU}}$ is a monotonic increasing function of $\tau_{\mathrm{ECVT}}$. In order to achieve the Type I.I power flow (7) must be used to calculate the gear ratios $k_{1}$ and $k_{2}$ of the planetary gear trains.

If $\tau_{\mathrm{ECVT}, \mathrm{eq}}$ is a decreasing function of $\tau_{\mathrm{ECVT}}$ a power flow of Type II is obtained in the Equivalent e-CVT (Figure 4(c)); that is, the power through the e-CVT is greater than the input power of the Equivalent e-CVT. Hence, with a monotonic increase of $\tau_{\mathrm{CVU}}$ as a function of $\tau_{\mathrm{ECVT} \text {,eq }}$ (Figure $4(\mathrm{~b})$ ) a power flow named Type I.II (Figure $4(\mathrm{a})$ ) is obtained by imposing that $\tau_{\mathrm{CVU}}$ is a decreasing function of $\tau_{\mathrm{ECVT}}$. Therefore, Type I.II power flow is obtained solving (8) to find the parameters $k_{1}$ and $k_{2}$ of the planetary gear trains.

If $\tau_{\mathrm{CVU}}$ is a decreasing function $\tau_{\mathrm{ECVT} \text {,eg }}$ (Figure 5(b)), the Equivalent e-CVT is in the Input Split (IS) configuration (Figure 5(c)) and Type I power flow occurs when $\tau_{\mathrm{ECVT} \text {,eq }}$ is an increasing function of $\tau_{\text {ECVT }}$. Therefore, considering the whole transmission, a Type II.I power flow occurs if $\tau_{\mathrm{CVU}}$ is a decreasing function of $\tau_{\mathrm{ECVT}}$ (Figure 5(a)). Hence, Type II.I power flow is achieved when (8) are solved to obtain $k_{1}$ and $k_{2}$.

If $\tau_{\mathrm{CVU}}$ is a decreasing function of $\tau_{\mathrm{ECVT} \text {,eq }}$ and $\tau_{\mathrm{ECVT} \text {,eq }}$ is a decreasing function of $\tau_{\mathrm{ECVT}}$, Type II.II power flow occurs in the Output Compound Split (Figure 6(a)). Then, the power flow in Figure 6(a) occurs when $\tau_{\mathrm{CVU}}$ increases with increasing $\tau_{\text {ECVT }}$.

In conclusion, with (7), Type I.I and Type II.II are the two possible power flows that occur in the transmission. On the contrary, Type I.II and Type II.I power flows are achieved with (8). 


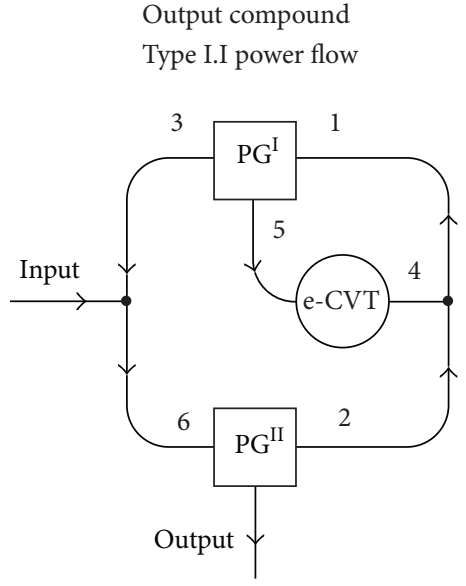

(a)

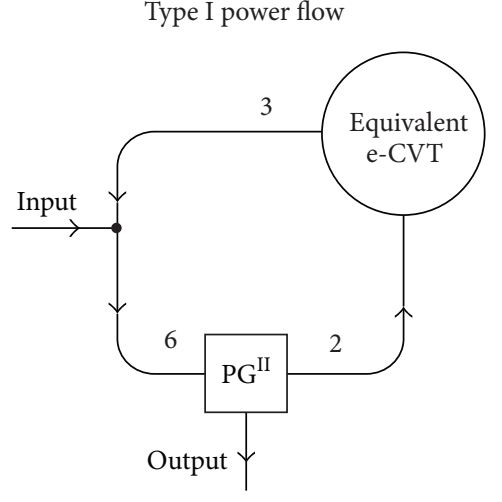

(b)

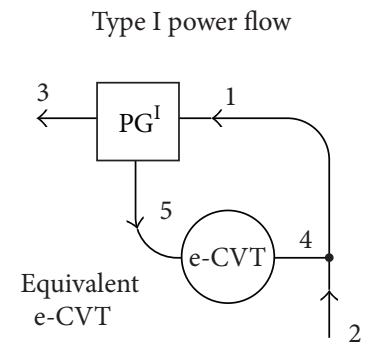

(c)

FIgure 3: Power flows in a Compound Split e-CVT with Type I.I power flow: (a) Output Compound Split e-CVT, (b) The Output Split with Equivalent e-CVT and Type I power flow, and (c) the Equivalent e-CVT with Type I power flow.

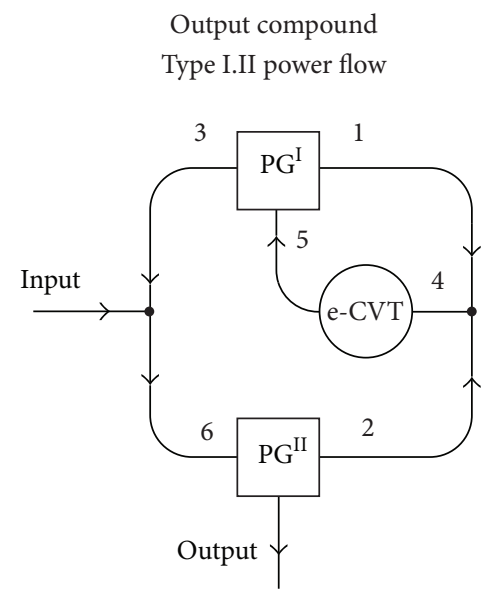

(a)

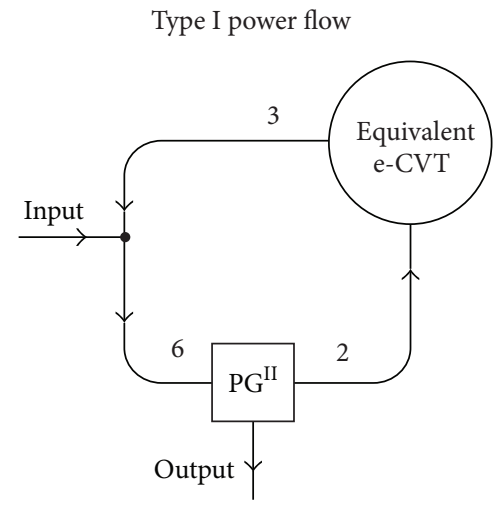

(b)

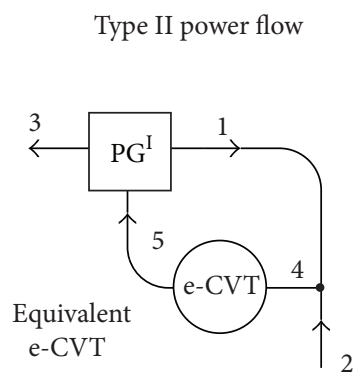

(c)

FIgure 4: Power flows in a Compound Split e-CVT with Type I.II power flow: (a) Output Compound Split e-CVT, (b) the Output Split with Equivalent e-CVT and Type I power flow, and (c) the Equivalent e-CVT with Type II power flow.

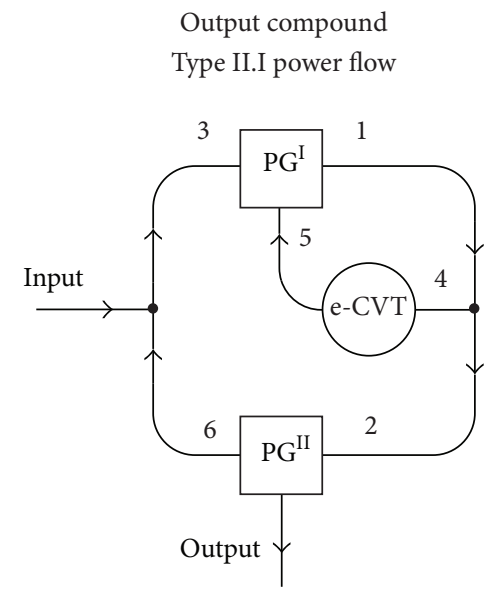

(a)

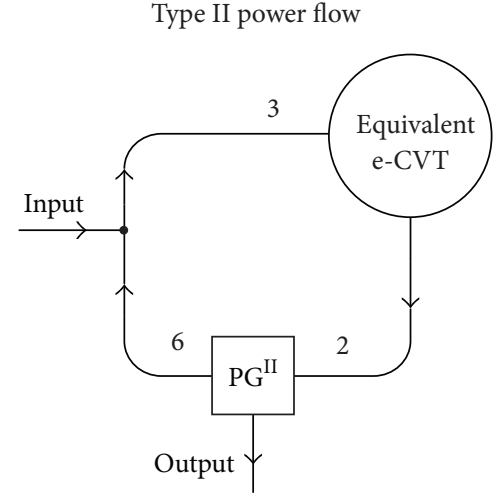

(b)
Type I power flow

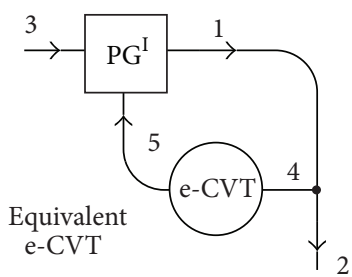

(c)

FIGURE 5: Power flows in a Compound Split e-CVT with Type II.I power flow: (a) Output Compound Split e-CVT, (b) the Output Split with Equivalent e-CVT and Type II power flow, and (c) the Equivalent e-CVT with Type I power flow. 


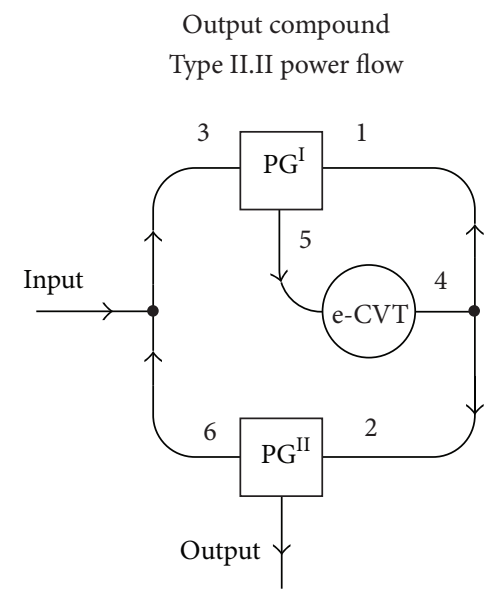

(a)

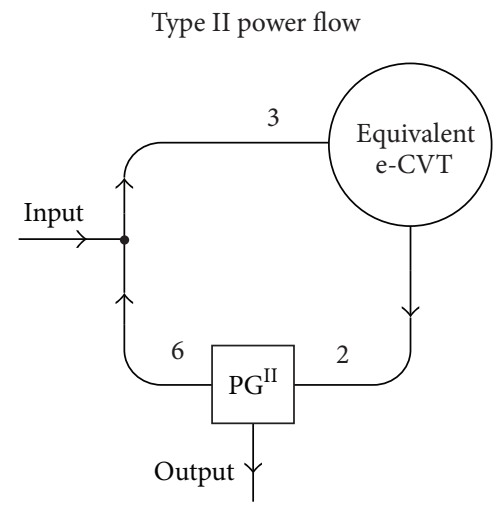

(b)

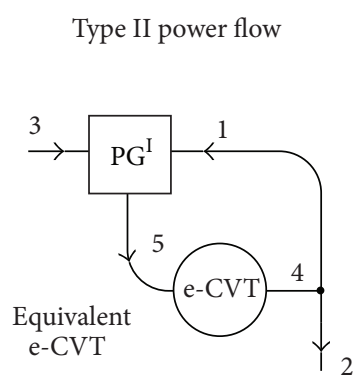

(c)

FIgure 6: Power flows in a Compound Split e-CVT with Type II.II power flow: (a) Output Compound Split e-CVT, (b) the Output Split with Equivalent e-CVT and Type II power flow, and (c) the Equivalent e-CVT with Type II power flow.

Finally, in agreement with the considerations suggested in [21], if the following inequality is verified

$$
\frac{\left(\tau_{\mathrm{CVU} M}-1\right)}{\left(\tau_{\mathrm{CVU} m}-1\right)}<\frac{\left(\tau_{\mathrm{ECVT} M}-1\right)}{\left(\tau_{\mathrm{ECVT} m}-1\right)}
$$

the Type I.I or Type II.I are the working conditions of the transmission. On the contrary, if

$$
\frac{\left(\tau_{\mathrm{CVU} M}-1\right)}{\left(\tau_{\mathrm{CVU} m}-1\right)}>\frac{\left(\tau_{\mathrm{ECVTM}}-1\right)}{\left(\tau_{\mathrm{ECVT} m}-1\right)}
$$

the Type I.II or Type II.II are established in the CVU.

For the efficiency of Output Compound Split Transmission the approach is based upon the assumption that only the losses in the e-CVT are considered. This assumption is generally accepted because the efficiency $\eta_{\mathrm{ECVT}}$ of the eCVT is very low (e.g., an electric motor-generator) compared with the other components. This condition is named the "real system."

The Output Compound Split Transmission with power flow of Type I.I is considered (Figure 3(a)). Both the ideal system (without losses) and the real system are analyzed and compared to each other. For given kinematic conditions $\left(\omega_{i}\right.$, $\left.\tau_{\mathrm{ECVT}}\right)$ and output torque $T_{o}$, the ratios of the torques of $\mathrm{PG}^{\mathrm{II}}$ shaft (branches "2" and "6") are fixed, and so the ratios between powers are also determined. This means that the powers in the branches " 2 " $\left(P_{2}\right)$ and " 6 " $\left(P_{6}\right)$ have the same value in the ideal and the real system.

On the other hand, the input power and the power of branch 3 are different in the ideal system and in the real system. In the real system, because of the power loss of the e-CVT, the input power $P_{\text {in }}$ is increased and the power $P_{3}$ is decreased.
It has been demonstrated in [28] that the ratio given by the power through the e-CVT branch divided by the input power of the overall CVU can be easily calculated (under the assumption of negligible power loss) relying only on the system kinematics:

$$
\left|\frac{P_{\mathrm{ECVT}}}{P_{\text {in }}}\right|_{\text {ideal }}=\left|\frac{d \tau_{\mathrm{CVU}}}{d \tau_{\mathrm{ECVT}}} \frac{\tau_{\mathrm{ECVT}}}{\tau_{\mathrm{CVU}}}\right| .
$$

Equation (15) is general and, therefore, it can be applied to the Compound Split Transmission as well as the Equivalent eCVT. As mentioned above, the input power $P_{2}$ (Figure 3(c)) of the Equivalent e-CVT does not change when the ideal and the real cases are compared. Hence, (15) is suitable for calculating the power in the real system. The power ratio is simply given by

$$
\begin{aligned}
\left|\frac{P_{\mathrm{ECVT}, \mathrm{eq}}}{P_{\text {in }}}\right|_{\text {ideal }} & =\left|\frac{P_{2}}{P_{\text {out }}}\right|_{\text {ideal }}=\left|\frac{P_{2}}{P_{\text {out }}}\right|_{\text {real }} \\
& =\left|\frac{d \tau_{\mathrm{CVU}}}{d \tau_{\mathrm{ECVT}, \mathrm{eq}}} \frac{\tau_{\mathrm{ECVT}, \mathrm{eq}}}{\tau_{\mathrm{CVU}}}\right| \\
& =\left|\frac{1+k_{2}+k_{1}+k_{2} k_{1}}{1+k_{1}+k_{2}\left(1-\tau_{\mathrm{ECVT}}\right)}\right| .
\end{aligned}
$$

The power loss in the Equivalent e-CVT of the real system can be obtained as follows:

$$
\begin{aligned}
P_{w_{\mathrm{CVU}}} & =-\left(1-\eta_{\mathrm{ECVT}, \mathrm{eq}}\right)\left|P_{2}\right| \\
& =-\left(1-\eta_{\mathrm{ECVT}, \mathrm{eq}}\right)\left|P_{\mathrm{ECVT}, \mathrm{eq}}\right|
\end{aligned}
$$


Using (16)-(17) and the power at the input shaft of the CVU, the efficiency can be finally calculated:

$$
\begin{aligned}
\eta_{\mathrm{CVU}} & =-\frac{P_{\text {out }}}{P_{\text {in }}}=\frac{P_{\text {out }}}{P_{\text {out }}+P_{w_{\mathrm{CVU}}}} \\
& =\frac{1}{1+\left(1-\eta_{\mathrm{ECVT}, \mathrm{eq}}\right)\left|P_{\mathrm{ECVT}, \mathrm{eq}} / P_{\text {in }}\right|_{\text {real }}} \\
& =\frac{1}{1+\left(1-\eta_{\mathrm{ECVT}, \mathrm{eq}}\right)\left|P_{\mathrm{ECVT}, \mathrm{eq}} / P_{\text {in }}\right|_{\text {ideal }}} .
\end{aligned}
$$

The efficiency $\eta_{\mathrm{ECVT} \text {,eq }}$ of Equivalent e-CVT can be derived using the same approach.

Indeed, if the Equivalent e-CVT is considered (Figure 3(c)), with given $\omega_{2}, \tau_{\mathrm{ECVT}}$, and $T_{3}$, the angular velocities and torques at branches " 1 " and " 5 " do no differ between the ideal and real system.

The power loss in the e-CVT of the real Equivalent ECVT can be easily written as a function of the power $P_{5}$ :

$$
P_{w_{\mathrm{ECVT}}}=-\left(1-\eta_{\mathrm{ECVT}}\right)\left|P_{5}\right| .
$$

Therefore, it follows that the efficiency in the Equivalent eCVT is

$$
\begin{aligned}
\eta_{\mathrm{ECVT}, \mathrm{eq}} & =-\frac{P_{\text {out }}}{P_{\text {in }}}=\frac{P_{\text {out }}}{P_{\text {out }}+P_{w_{\mathrm{ECVT}}}}=\frac{1}{1+P_{w_{\mathrm{ECVT}}} / P_{\mathrm{out}}} \\
& =\frac{1}{1+\left(1-\eta_{\mathrm{ECVT}}\right)\left|P_{5} / P_{3}\right|_{\text {ideal }}} \\
& =\frac{1}{1+\left(1-\eta_{\mathrm{ECVT}}\right)\left|P_{\mathrm{ECVT}} / P_{3}\right|_{\text {ideal }}} .
\end{aligned}
$$

By considering (11) and (15) the power ratio in the Equivalent e-CVT can be obtained as follows (Figure 3(c)):

$$
\begin{aligned}
\left|\frac{P_{\mathrm{ECVT}}}{P_{2}}\right|_{\text {ideal }} & =\left|\frac{P_{\mathrm{ECVT}}}{P_{3}}\right|_{\text {ideal }}=\left|\frac{P_{5}}{P_{3}}\right|_{\text {ideal }} \\
& =\left|\frac{\partial \tau_{\mathrm{ECVT}, \mathrm{eq}}}{\partial \tau_{\mathrm{ECVT}}} \frac{\tau_{\mathrm{ECVT}}}{\tau_{\mathrm{ECVT}, \mathrm{eq}}}\right|=\left|\frac{\tau_{\mathrm{ECVT}}}{\tau_{\mathrm{ECVT}}+k_{1}}\right| .
\end{aligned}
$$

In conclusion, the efficiency can be calculated with (18) in which $\eta_{\text {ECVT,eq }}$ can be obtained by (20), where $\mid P_{\text {ECVT,eq }} /$ $\left.P_{\text {in }}\right|_{\text {ideal }}$ and $\left|P_{\mathrm{ECVT}} / P_{3}\right|_{\text {ideal }}$ are derived from (16) and (21), respectively.

For the Output Compound Split Transmission with power flow of Type I.II, the CVU architecture is the same as the previous case (Figure 4(b)). Therefore, the power loss is simply given by (17) and the efficiency by (18), whereas the formula for $\eta_{\mathrm{ECVT}}$,eq is different.

The power flow in the Equivalent e-CVT and its efficiency $\eta_{\text {ECVT,eq }}$ change compared with the previous case. Also, in this case, $P_{5}$ does not change when comparing the ideal system and the real system (Figure $4(\mathrm{c})$ ). This means that the expression for the power loss in the Equivalent e-CVT is a function of the e-CVT output power $P_{5}$; that is, the power loss is given by

$$
P_{w_{\mathrm{CVT}, \mathrm{eq}}}=-\frac{\left(1-\eta_{\mathrm{ECVT}}\right)}{\eta_{\mathrm{ECVT}}}\left|P_{5}\right|
$$

From (20) and (22), the efficiency of the Equivalent e-CVT is

$$
\begin{aligned}
\eta_{\mathrm{ECVT}, \mathrm{eq}} & =-\frac{P_{\mathrm{out}}}{P_{\mathrm{in}}}=\frac{P_{\mathrm{out}}}{P_{\mathrm{out}}+P_{w_{\mathrm{ECVT}}}}=\frac{1}{1+P_{w_{\mathrm{ECVT}}} / P_{\mathrm{out}}} \\
& =\frac{1}{1+\left(\left(1-\eta_{\mathrm{ECVT}}\right) / \eta_{\mathrm{ECVT}}\right)\left|P_{\mathrm{ECVT}} / P_{3}\right|_{\text {ideal }}} .
\end{aligned}
$$

In conclusion, the efficiency of Type I.II power flow can be calculated through (18) where $\eta_{\mathrm{ECVT} \text {,eq }}$ can be obtained by (23).

The efficiency of the CVU working with Type II.I power flow is derived using a similar approach.

From Figure 5(b) it follows that the power $P_{2}$ which has the same value in the ideal system and in the real system is the output power of the Equivalent e-CVT. The power loss in the e-CVT of the real Equivalent e-CVT (Figure 4(c)) is

$$
P_{w_{\mathrm{CVU}}}=-\frac{\left(1-\eta_{\mathrm{ECVT}, \mathrm{eq}}\right)}{\eta_{\mathrm{ECVT}, \mathrm{eq}}}\left|P_{2}\right| .
$$

Thus, the efficiency of the CVU is given by

$$
\begin{gathered}
\eta_{\mathrm{CVU}}=-\frac{P_{\mathrm{out}}}{P_{\mathrm{in}}}=\frac{P_{\mathrm{out}}}{P_{\mathrm{out}}+P_{w_{\mathrm{CVU}}}} \\
=\frac{1}{1+\left(\left(1-\eta_{\mathrm{ECVT}, \mathrm{eq}}\right) / \eta_{\mathrm{ECVT}, \mathrm{eq}}\right)\left|P_{\mathrm{ECVT}, \mathrm{eq}} / P_{\text {in }}\right|_{\text {ideal }}} .
\end{gathered}
$$

It follows that the power loss and efficiency of the Equivalent e-CVT are given by

$$
\begin{aligned}
P_{w_{\mathrm{ECVT}, \mathrm{eq}}} & =-\frac{\left(1-\eta_{\mathrm{ECVT}}\right)}{\eta_{\mathrm{ECVT}}}\left|P_{5}\right|, \\
\eta_{\mathrm{ECVT}, \mathrm{eq}} & =-\frac{P_{\mathrm{out}}}{P_{\mathrm{in}}}=\frac{P_{\mathrm{in}}+P_{w_{\mathrm{ECVT}}}}{P_{\mathrm{in}}} \\
& =1-\frac{\left(1-\eta_{\mathrm{ECVT}}\right)}{\eta_{\mathrm{ECVT}}}\left|\frac{P_{\mathrm{ECVT}}}{P_{2}}\right|_{\text {ideal }} .
\end{aligned}
$$

The power ratio in the Equivalent e-CVT is obtained using (21); hence, the efficiency in the Equivalent e-CVT follows from (27).

In conclusion, the efficiency of Type II.I can be calculated through (25) where $\eta_{\text {ECVT,eq }}$ can be obtained by (27).

Finally, in the Output Compound Split Transmission with power flow of Type II.II we obtained (Figure 6)

$$
P_{w_{\mathrm{ECVT}, \mathrm{eq}}}=-\left(1-\eta_{\mathrm{ECVT}}\right)\left|P_{5}\right| \text {. }
$$


The power ratio in the Equivalent e-CVT follows from (21). Therefore, the efficiency of the Equivalent e-CVT is deduced from equation

$$
\begin{aligned}
\eta_{\mathrm{ECVT}, \mathrm{eq}} & =-\frac{P_{\mathrm{out}}}{P_{\mathrm{in}}}=\frac{P_{\mathrm{in}}+P_{w_{\mathrm{ECVT}}}}{P_{\mathrm{in}}} \\
& =1-\left(1-\eta_{\mathrm{ECVT}}\right)\left|\frac{P_{\mathrm{ECVT}}}{P_{2}}\right|_{\text {ideal }} .
\end{aligned}
$$

It can be noticed that the power flow of Figure 6(b) is identical to Figure 5(b). It follows that also in this case the efficiency is calculated with (25), with $\eta_{\mathrm{ECVT} \text {,eq }}$ calculated as (29).

\section{Numerical Example}

In this section a numerical example is given, which also includes the calculation of the transmission efficiency, using the simplified approach described in this work. The calculations can be performed after the speed ratio of the CVU and the e-CVT have been imposed. It is here assumed that speed ratio of the e-CVT varies between the bounds: $\tau_{\mathrm{ECVT} m}=0.4$ and $\tau_{\text {ECVTM }}=2$, which is typical of mechanical CVT. The ratio range of the $\mathrm{CVU}$ is a system requirement, so it can be chosen arbitrarily. A CVU is here designed, with a speed ratio bounded between $\tau_{\mathrm{CVU} m}=-1$ and $\tau_{\mathrm{CVUM}}=2$, in order to comprise also the neutral gear. These CVU bounds can be obtained only by choosing two planetary gear trains characterized by $k_{1}$ and $k_{2}$ calculated with (7), in the case of monotonic increase of the CVU speed ratio for increasing ECVT speed ratio. It is obtained that

$$
\begin{aligned}
& k_{1}=0.29 \\
& k_{2}=-3.29
\end{aligned}
$$

If the case of a monotonic decreasing CVU speed ratio with increasing e-CVT speed ratio, $k_{1}$ and $k_{2}$ must be calculated with (8), it follows that

$$
\begin{aligned}
& k_{1}=-10 \\
& k_{2}=-17 .
\end{aligned}
$$

The CVU speed ratio as a function of the e-CVT speed ratio given by (4) is shown in Figure 7 for the two aforementioned cases.

The power ratio $\left|P_{\mathrm{ECVT}} / P_{\mathrm{CVU}}\right|$, calculated with (15), is shown in Figure 8.

Two regions can be distinguished, corresponding to two different ranges of $\tau_{\mathrm{CVU}}$ : the first range $0 \leq \tau_{\mathrm{CVU}} \leq 2$ and the second range $-1<\tau_{\mathrm{CVU}}<0$.

Each region corresponds to a different power circulation. Considering the limits imposed on the speed ratio of this example, it results in the following:

$$
\frac{\left(\tau_{\mathrm{CVU}_{M}}-1\right)}{\left(\tau_{\mathrm{CVU}_{m}}-1\right)}>\frac{\left(\tau_{\mathrm{ECVT}_{M}}-1\right)}{\left(\tau_{\mathrm{ECVT}_{m}}-1\right)} .
$$

With the help of (13) and (14), one can determine the power flow type, taking also into account the sign change of the

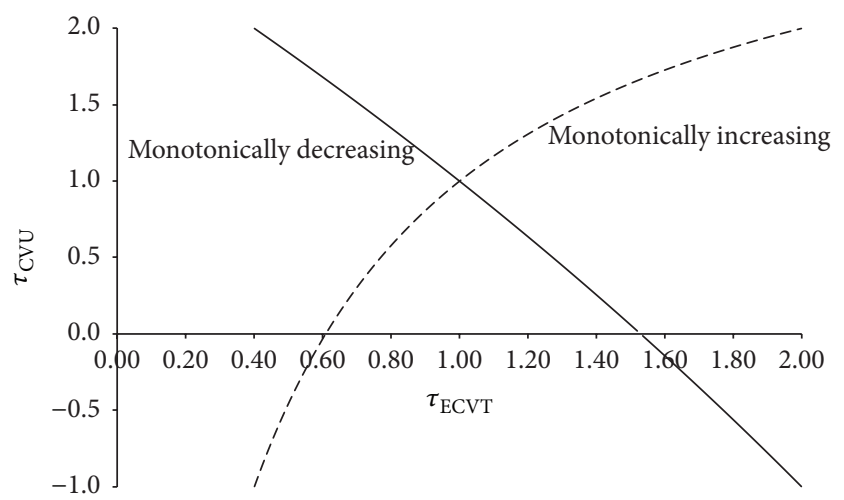

FIGURE 7: Speed ratio of the CVU $\left(\tau_{\mathrm{CVU}}\right)$ as a function of the e-CVT speed ratio $\left(\tau_{\mathrm{ECVT}}\right)$.

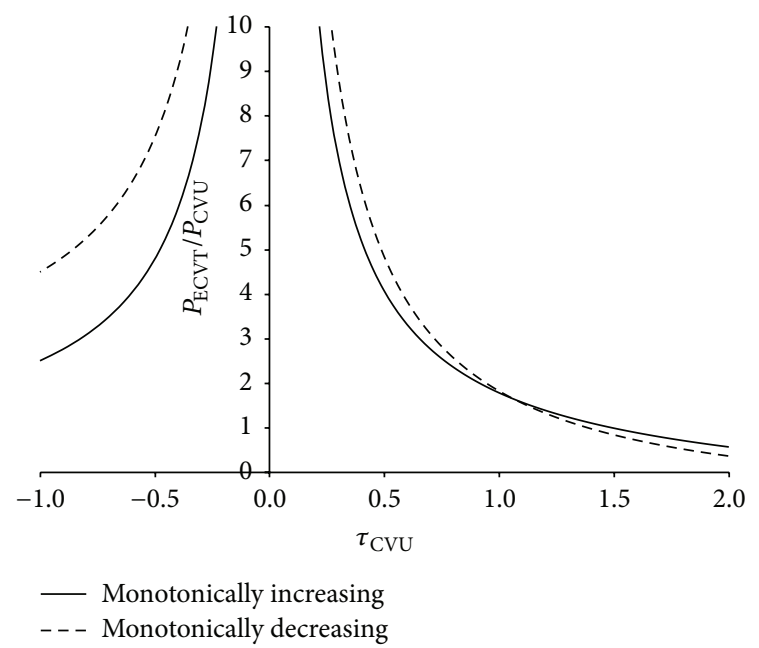

Figure 8: Power ratio $\left|P_{\mathrm{ECVT}} / P_{\mathrm{CVU}}\right|$ considering no losses plotted as a function of the overall speed ratio $\tau_{\mathrm{CVU}}$.

ratio $\tau_{\mathrm{CVU}}$. In fact, when $\tau_{\mathrm{CVU}}$ is an increasing function of $\tau_{\text {ECVT }}$, the Type II.II power flow occurs for $\tau_{\mathrm{CVU}}>0$, as a consequence of (14), whereas, in the other region with $\tau_{\mathrm{CVU}}<$ 0 , the power flow changes from Type II.II to Type I.II [21].

On the other hand, when $\tau_{\mathrm{CVU}}$ is a decreasing function of $\tau_{\mathrm{ECVT}},(14)$ still holds, but in this case the Type I.II power flow occurs if $\tau_{\mathrm{CVU}}>0$, whereas if $\tau_{\mathrm{CVU}}<0$, then the power flow is changed from Type I.II to Type II.II [21].

The ratio given by the power through the e-CVT divided by the global power is not equal in the two cases (Figure 8). With $\tau_{\mathrm{CVU}}<1$ the power ratio is larger when the speed ratio of the transmission is a decreasing function of the speed ratio of the e-CVT. Only with $\tau_{\mathrm{CVU}}>1$ the situation is the opposite one.

The efficiency $\eta_{\mathrm{CVU}}$ plotted as a function of $\tau_{\mathrm{CVU}}$ is shown in Figure 9, considering that $\eta_{\mathrm{ECVT}}=0.9$. For $\tau_{\mathrm{CVU}}>$ 0 and with a monotonically increasing CVU speed ratio with increasing e-CVT speed ratio (power flow of Type II.II), (16), (21), (25), and (29) are utilized. In this case, the efficiency is larger than in the case of monotonically decreasing speed 


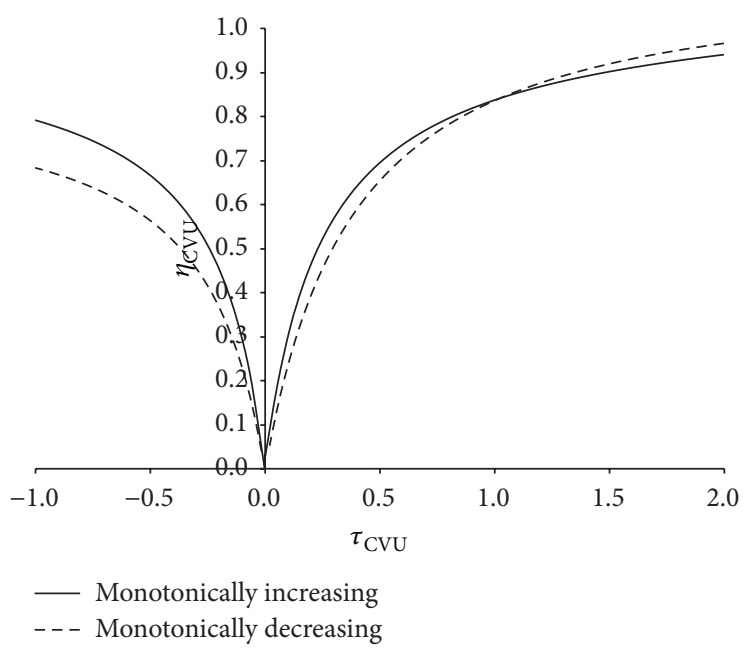

FIGURE 9: The efficiency $\eta_{\mathrm{CVU}}$ as a function of the overall speed ratio $\tau_{\mathrm{CVU}}\left(\eta_{\mathrm{ECVT}}=0.9\right)$.

ratio for $\tau_{\mathrm{CVU}}<1$, whereas the opposite condition is verified for $\tau_{\mathrm{CVU}}>1$. This is expected since the device with the lowest and most critical efficiency is the e-CVT, so the configuration that minimizes the power through the e-CVT is the most convenient one in terms of energy efficiency.

\section{Efficiency of the Output Compound e-CVT in Direct and Reverse Operation}

Hybrid vehicles are the main application for Compound Split-eCVT transmission. One of the most important features of hybrid vehicles is the regenerative braking capability. Such a function needs the transmission to work in reverse operation. For this reason it is important to evaluate the efficiency of the transmission, in both direct and reverse operation and to understand the differences.

In this section the same transmission data are considered $\left(\tau_{\mathrm{ECVT} m}=0.4, \tau_{\mathrm{ECVTM}}=2 ; \tau_{\mathrm{CVU} m}=-1, \tau_{\mathrm{CVU} M}=2\right)$ and the performance in reverse operation are calculated through the model developed in a previous work [22]. The efficiency $\eta_{\text {ECVT }}$ of the e-CVT is considered constant and given by $\eta_{\text {ECVT }}=0.9$. As mentioned, in the compound transmission with a monotonic increasing speed ratio $\left(k_{1}=0.29 ; k_{2}=\right.$ -3.29), if $\tau_{\mathrm{CVUm}}>0$, a power flow of Type II.II is obtained. Given the same kinematic parameters, when the transmission works in reverse operation (Figure 10) the input and output torques are reversed and, because of the planetary gear train $\mathrm{PG}^{1}$, also the torques on links 2 and 6 are reversed. Furthermore, the torque and the power on the link 3 are also reversed and, as a consequence of the planetary gear train $\mathrm{PG}^{2}$, also the torque and power on links 1, 4, and 5 are reversed. So, in the change from direct operation (Figure 10(a)) to reverse operation (Figure 10(b)), the power flow is reversed on all the links and the power flow is yet of Type II.II.

When $\tau_{\mathrm{CVU}}<0$, similar arguments leads to conclude that the power flow is of Type I.II also in reverse operation (Figure 11).
The monotonic increasing speed ratio is investigated first. In Figure 12, it is shown that in reverse operation the efficiency of the transmission is always less than in direct operation, with the only exception of the high values of the speed ratio $\left(\tau_{\mathrm{CVU}}>1.7\right)$. It is very interesting to see that when the speed ratio is close to zero $\left(-0.27<\tau_{\mathrm{CVU}}<0.22\right)$, the efficiency in reverse operation is negative, which means that the transmission is nonreversible with the speed ratio in the aforementioned range. This result is in agreement with the results shown in [8] in the case of Power Split Transmissions. It is actually relevant for applications to the hybrid vehicles, because it affects negatively the braking recovery capabilities. In particular, it is not possible to recover energy in braking with $-0.27<\tau_{\mathrm{CVU}}<0.22$.

Similar results can be obtained in the case of monotonic decreasing speed ratio (Figure 7). Given the speed ratio ranges of CVT and CVU (which give $k_{1}=-10$ and $k_{2}=$ -17 ), also in this case, the power flows do not change when switching from direct to reverse operation: power flow of Type I.II with $\tau_{\mathrm{CVU}}>0$ (Figure 11) and power flow of Type II.II with $\tau_{\mathrm{CVU}}<0$ (Figure 10). The efficiency in direct and reverse operation is shown in Figure 13. Also in this case the efficiency in reverse operation is visibly less than in direct mode. Furthermore, the nonreversibility range of the speed is even larger, being $-0.40<\tau_{\mathrm{CVU}}<0.28$.

\section{Conclusions}

In recent years, compound transmissions are attracting increasing interest for application in hybrid vehicles, because they are flexible and they permit optimizing the efficiency in different operating conditions. The goal of this work was to obtain a systematic approach to determine the power flows and the efficiency in Output Compound Split Transmissions, for optimal design and control of HEVs. The proposed methodology is suitable for steady-state working conditions, and it follows from the assumption of negligible power loss in all the components except for the e-CVT. It is shown how 


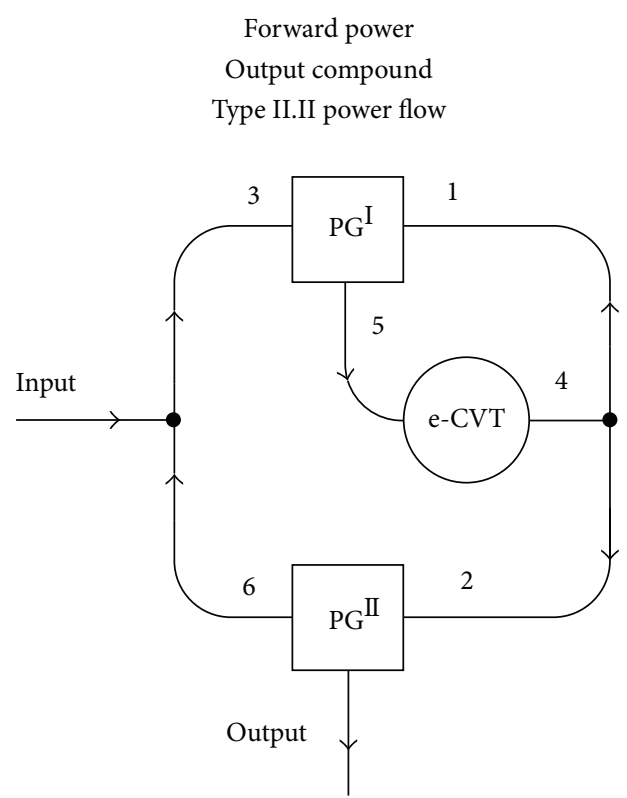

(a)

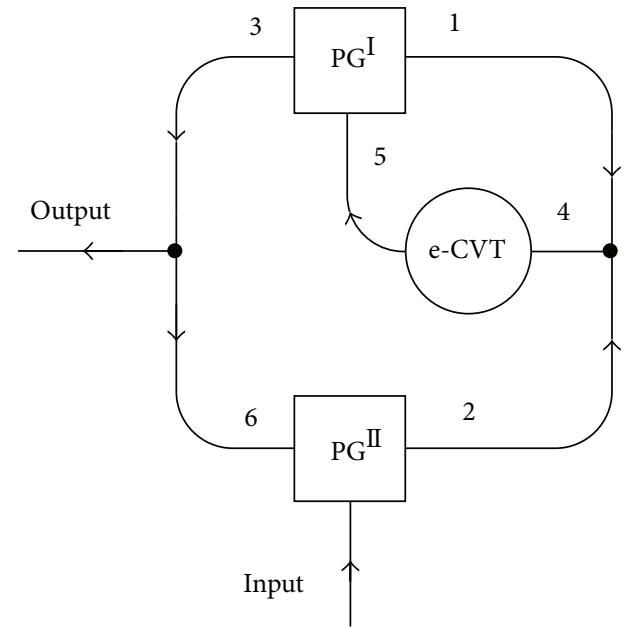

(b)

FIgure 10: Power flows in a Output Compound Split e-CVT with Type II.II power flow: (a) forward power, (b) reverse power.

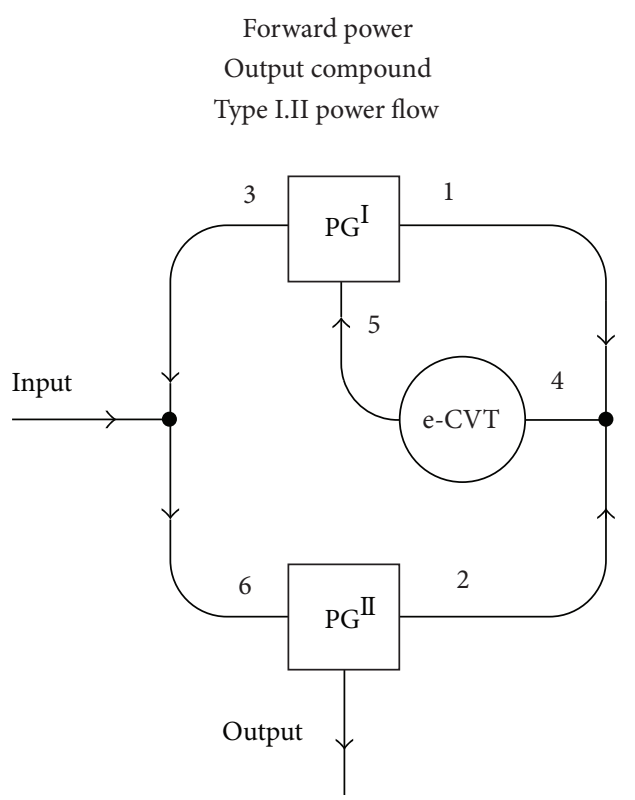

(a)

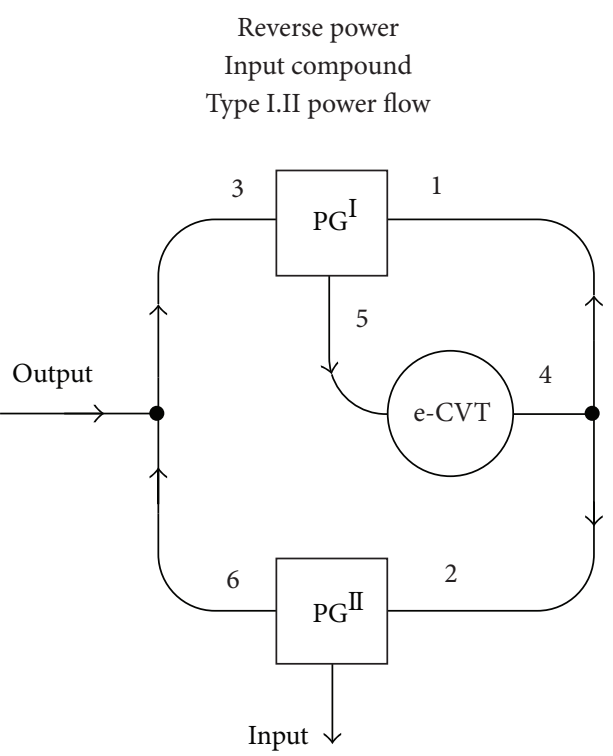

(b)

FIgUre 11: Power flows in a Output Compound Split e-CVT with Type I.II power flow: (a) forward power, (b) reverse power.

the power circulation depends only on kinematic conditions. Furthermore, a novel approach is shown to study the efficiency of the transmission, which relies on the separation of the compound transmission in two subsystems, so that it can be studied as an "Equivalent e-CVT" (which is a power split-like transmission of Input-Output Split type), inside an Output Split device. Following our approach, the efficiency is calculated easily for each of all the possible power flows. A numerical example showed the potential of this tool for power flows and efficiency calculations of the Output Compound Split Transmission, in direct and reverse operation. Experimental validation of the presented methodology is in progress and it will be the subject matter of future works. 


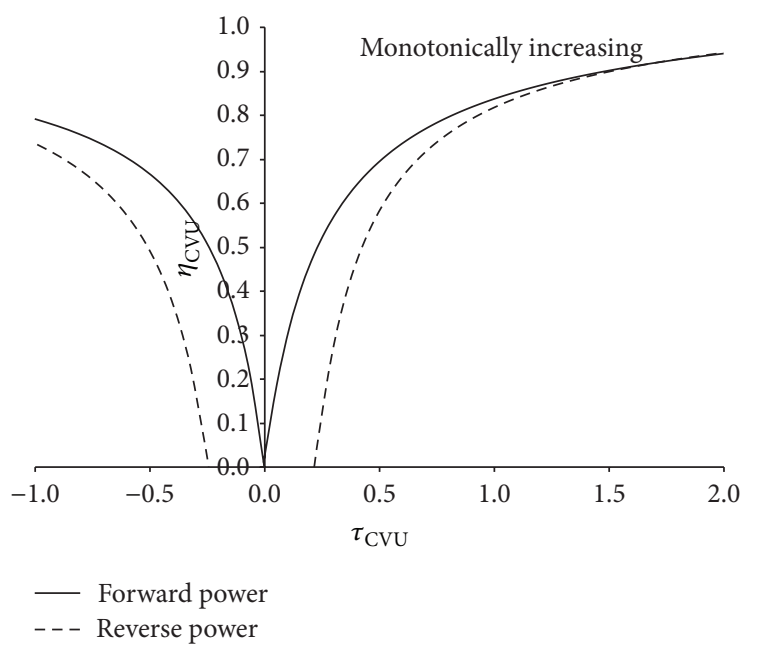

FIGURE 12: The efficiency $\eta_{\mathrm{CVU}}$ as a function of the overall speed ratio $\tau_{\mathrm{CVU}}\left(\eta_{\mathrm{ECVT}}=0.9\right)$, in forward and reverse power: transmission ratio monotonically increasing.

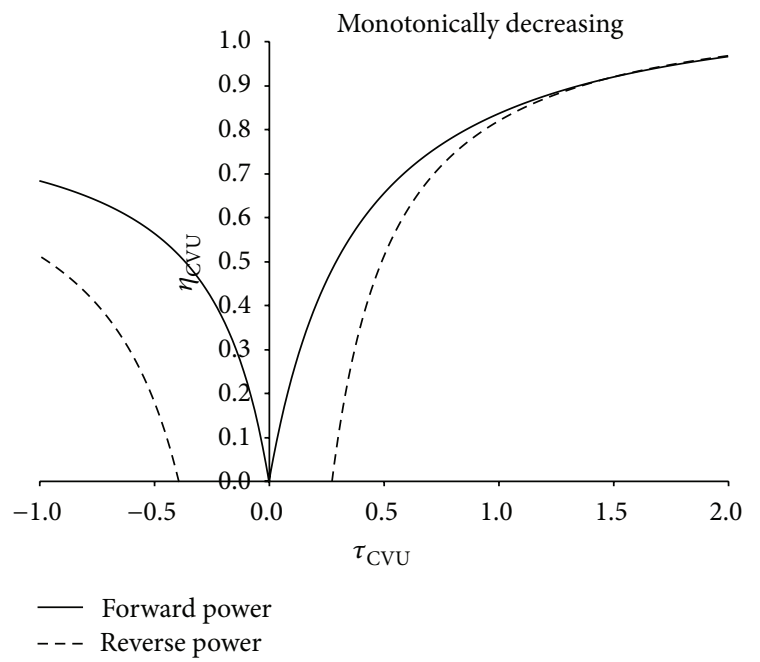

FIGURE 13: The efficiency $\eta_{\mathrm{CVU}}$ as a function of the overall speed ratio $\tau_{\mathrm{CVU}}\left(\eta_{\mathrm{ECVT}}=0.9\right)$, in forward and reverse power: transmission ratio monotonically decreasing.

\section{Nomenclature}

CVU, CVT, Continuously Variable Unit, Continuously ECVT, and PG Variable Transmission, Electronic (subscripts): Continuously Variable Transmission, and planetary gear

$M, m$, eq Maximum, minimum, and equivalent, (subscripts): respectively

$k_{1}, k_{2}$ : $\quad$ Kinematic parameter of the PG 1 and 2, respectively

$P_{j}: \quad \quad$ Power through the $j$ th branch

$P_{w}, P_{\text {in }}$, and Dissipated power, input power, and output

$P_{\text {out }}: \quad$ power

$\tau: \quad$ Speed ratio

$\eta: \quad$ Efficiency

$\omega_{j}$ : $\quad$ Angular velocity of the $j$ th shaft $\omega_{o}$ : Angular velocity of the output shaft of the CVU

$\omega_{i}$ : Angular velocity of the input shaft of the CVU.

\section{Conflict of Interests}

The authors declare that there is no conflict of interests regarding the publication of this paper.

\section{References}

[1] M. R. Cuddy and K. B. Wipke, "Analysis of the fuel economy benefit of drivetrain hybridization," SAE Technical Paper 970289, 1997.

[2] S. Stockar, V. Marano, M. Canova, G. Rizzoni, and L. Guzzella, "Energy-optimal control of plug-in hybrid electric vehicles for real-world driving cycles," IEEE Transactions on Vehicular Technology, vol. 60, no. 7, pp. 2949-2962, 2011.

[3] L. Serrao, S. Onori, and G. Rizzoni, "A comparative analysis of energy management strategies for hybrid electric vehicles," Journal of Dynamic Systems, Measurement and Control, vol. 133, no. 3, Article ID 031012, 2011.

[4] F. R. Salmasi, "Control strategies for hybrid electric vehicles: evolution, classification, comparison, and future trends," IEEE Transactions on Vehicular Technology, vol. 56, no. 5, pp. 23932404, 2007.

[5] F. Bottiglione, T. Contursi, A. Gentile, and G. Mantriota, "The fuel economy of hybrid buses: the role of ancillaries in real urban driving," Energies, vol. 7, no. 7, pp. 4202-4220, 2014.

[6] A. Taghavipour, N. L. Azad, and J. McPhee, "An optimal power management strategy for power split plug-in hybrid electric vehicles," International Journal of Vehicle Design, vol. 60, no. 3-4, pp. 286-304, 2012.

[7] Z. Zhao, C. Wang, T. Zhang, X. Dai, and X. Yuan, "Control optimization of a compound power-split hybrid transmission for electric drive," SAE Technical Papers, 2015.

[8] G. Mantriota, "Performances of a series infinitely variable transmission with type I power flow," Mechanism and Machine Theory, vol. 37, no. 6, pp. 579-597, 2002.

[9] G. Mantriota, "Performances of a parallel infinitely variable transmissions with a type II power flow," Mechanism and Machine Theory, vol. 37, no. 6, pp. 555-578, 2002.

[10] B. Si, "Reconfigurable Hybrid Gear Train," US Patent 0070992 A1, 2011.

[11] B. Si, "Dual mode input split compound split configuration EPPV transmission," U.S. Patent 8075435 B2, 2011.

[12] F. Bottiglione and G. Mantriota, "MG-IVT: an infinitely variable transmission with optimal power flows," Journal of Mechanical Design, vol. 130, no. 11, Article ID 112603, 2008.

[13] K. T. Chau and W. Li, "Overview of electric machines for electric and hybrid vehicles," International Journal of Vehicle Design, vol. 64, no. 1, pp. 46-71, 2014.

[14] A. G. Holmes and M. R. Schmidt, "Hybrid Electric Powertrain Including a Two-Mode Electrically Variable Transmission," US Patent 6478705 B1, 2002.

[15] C. Brendan, "Comparative analysis of single and combined hybrid electrically variable transmission operating modes," SAE Technical Paper 2005-01-1165, 2005.

[16] B. Mashadi and S. A. M. Emadi, "Dual-mode power-split transmission for hybrid electric vehicles," IEEE Transactions on Vehicular Technology, vol. 59, no. 7, pp. 3223-3232, 2010. 
[17] J. Kim, J. Kang, Y. Kim, T. Kim, B. Min, and H. Kim, "Design of power split transmission: design of dual mode power split transmission," International Journal of Automotive Technology, vol. 11, no. 4, pp. 565-571, 2010.

[18] Y. Zou, F.-C. Sun, C.-N. Zhang, and J.-Q. Li, "Optimal energy management strategy for hybrid electric tracked vehicles," International Journal of Vehicle Design, vol. 58, no. 2-4, pp. 307324, 2012.

[19] N.-D. Kim, J.-M. Kim, and H.-S. Kim, "Control strategy for a dual-mode electromechanical, infinitely variable transmission for hybrid electric vehicles," Proceedings of the Institution of Mechanical Engineers Part D: Journal of Automobile Engineering, vol. 222, no. 9, pp. 1587-1601, 2008.

[20] J. Kim, T. Kim, B. Min, S. Hwang, and H. Kim, "Mode control strategy for a two-mode hybrid electric vehicle using electrically variable transmission (EVT) and fixed-gear mode," IEEE Transactions on Vehicular Technology, vol. 60, no. 3, pp. 793-803, 2011.

[21] Z. Zhou, C. Mi, and G. Zhang, "Integrated control of electromechanical braking and regenerative braking in plug-in hybrid electric vehicles," International Journal of Vehicle Design, vol. 58, no. 2-4, pp. 223-239, 2012.

[22] F. Bottiglione and G. Mantriota, "Reversibility of power-split transmissions," Journal of Mechanical Design, Transactions of the ASME, vol. 133, no. 8, Article ID 084503, 2011.

[23] F. Bottiglione and G. Mantriota, "Effect of the ratio spread of CVU in automotive kinetic energy recovery systems," Journal of Mechanical Design, Transactions of the ASME, vol. 135, no. 6, Article ID 061001, 2013.

[24] F. Bottiglione, G. Carbone, L. De Novellis, L. Mangialardi, and G. Mantriota, "Mechanical hybrid KERS based on toroidal traction drives: an example of smart tribological design to improve terrestrial vehicle performance," Advances in Tribology, vol. 2013, Article ID 918387, 9 pages, 2013.

[25] S. De Pinto, F. Bottiglione, and G. Mantriota, "Infinitely variable transmissions in neutral gear: torque ratio and power recirculation," Mechanism and Machine Theory, vol. 74, pp. 285298, 2014.

[26] S. de Pinto and G. Mantriota, "A simple model for compound split transmissions," Proceedings of the Institution of Mechanical Engineers, Part D: Journal of Automobile Engineering, vol. 228, no. 5, pp. 549-564, 2014.

[27] G. Mantriota, "Fuel consumption of a vehicle with power split CVT system," International Journal of Vehicle Design, vol. 37, no. 4, pp. 327-342, 2005.

[28] M. Cammalleri, "Efficiency of split-way CVT's. A simplified model,” SAE Technical Papers 15, 2007. 

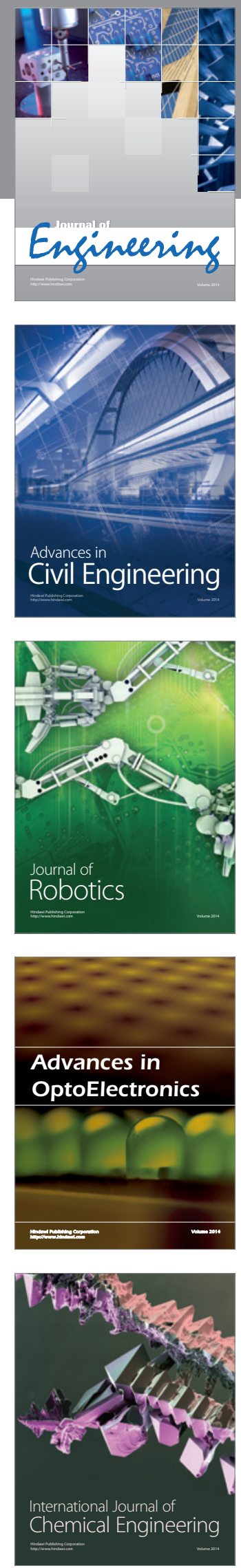

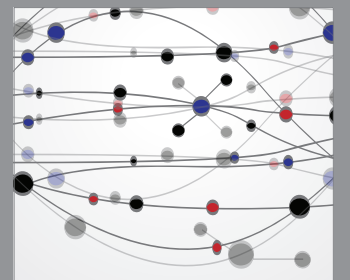

The Scientific World Journal
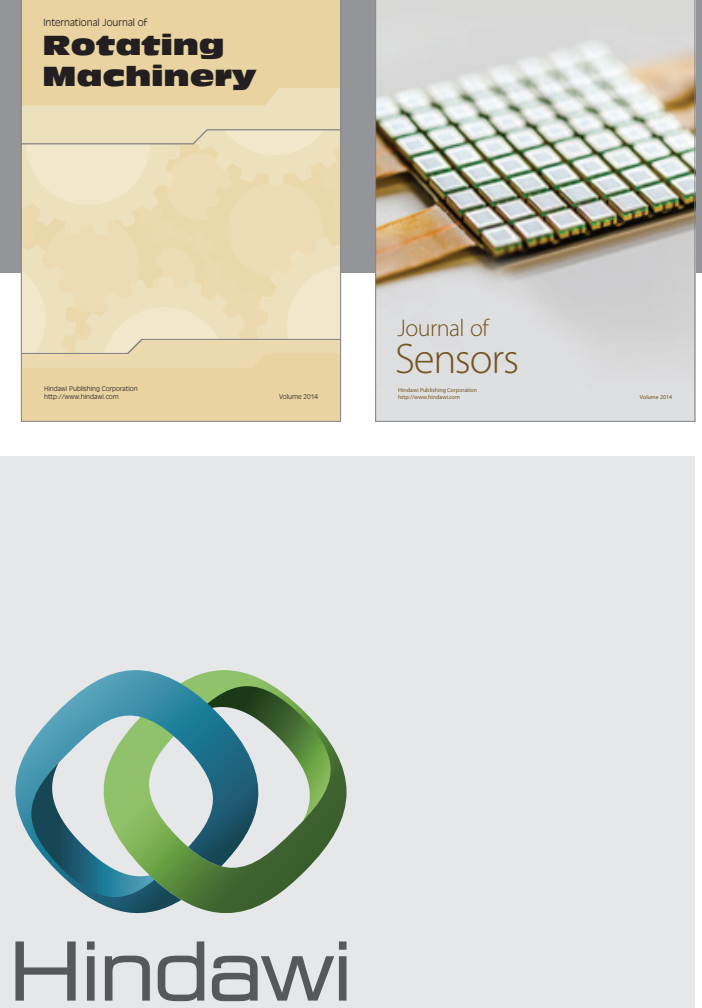

Submit your manuscripts at http://www.hindawi.com
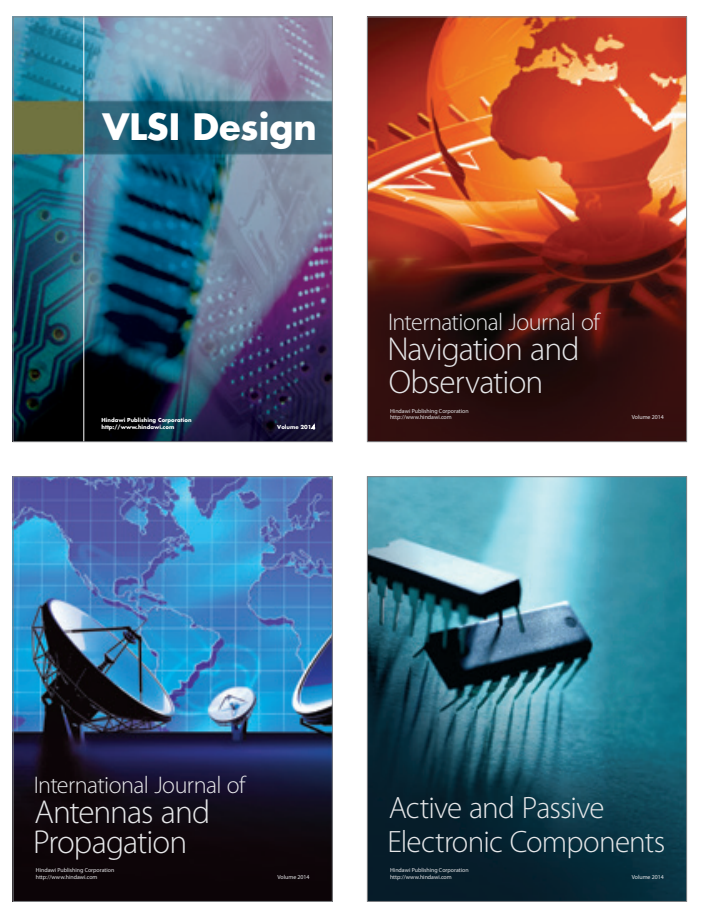
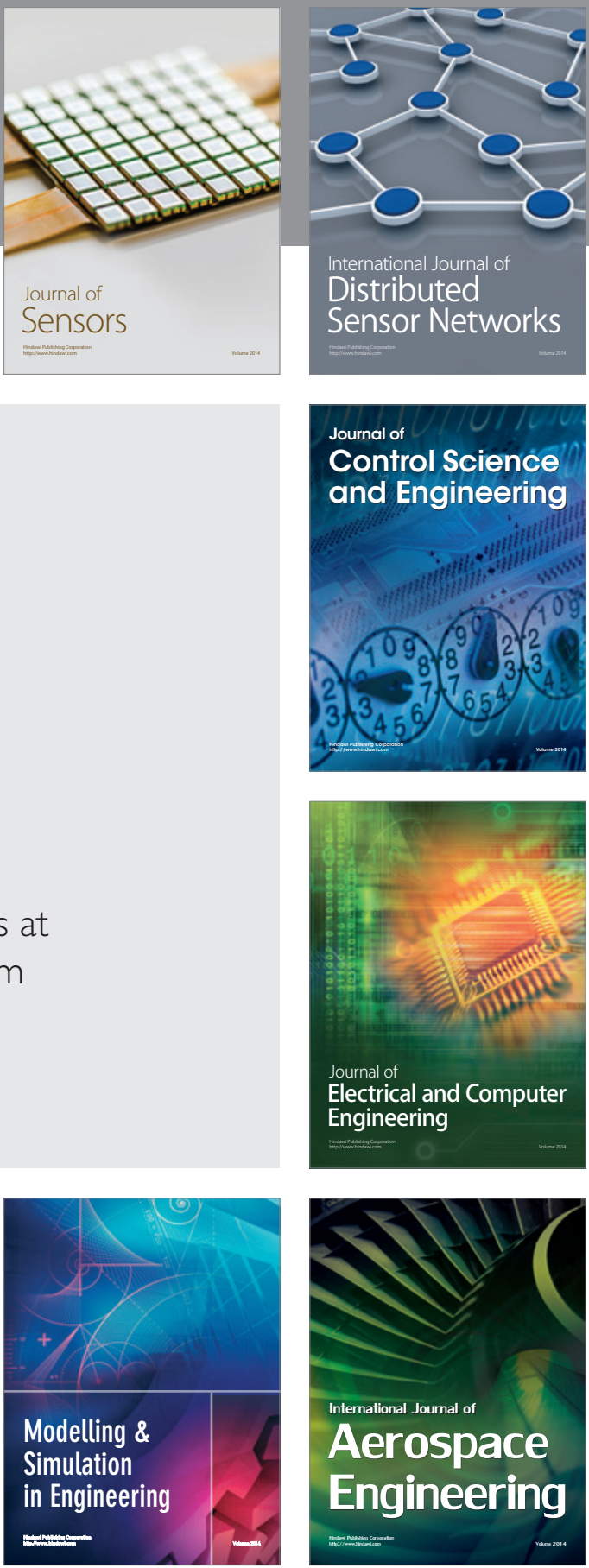

Journal of

Control Science

and Engineering
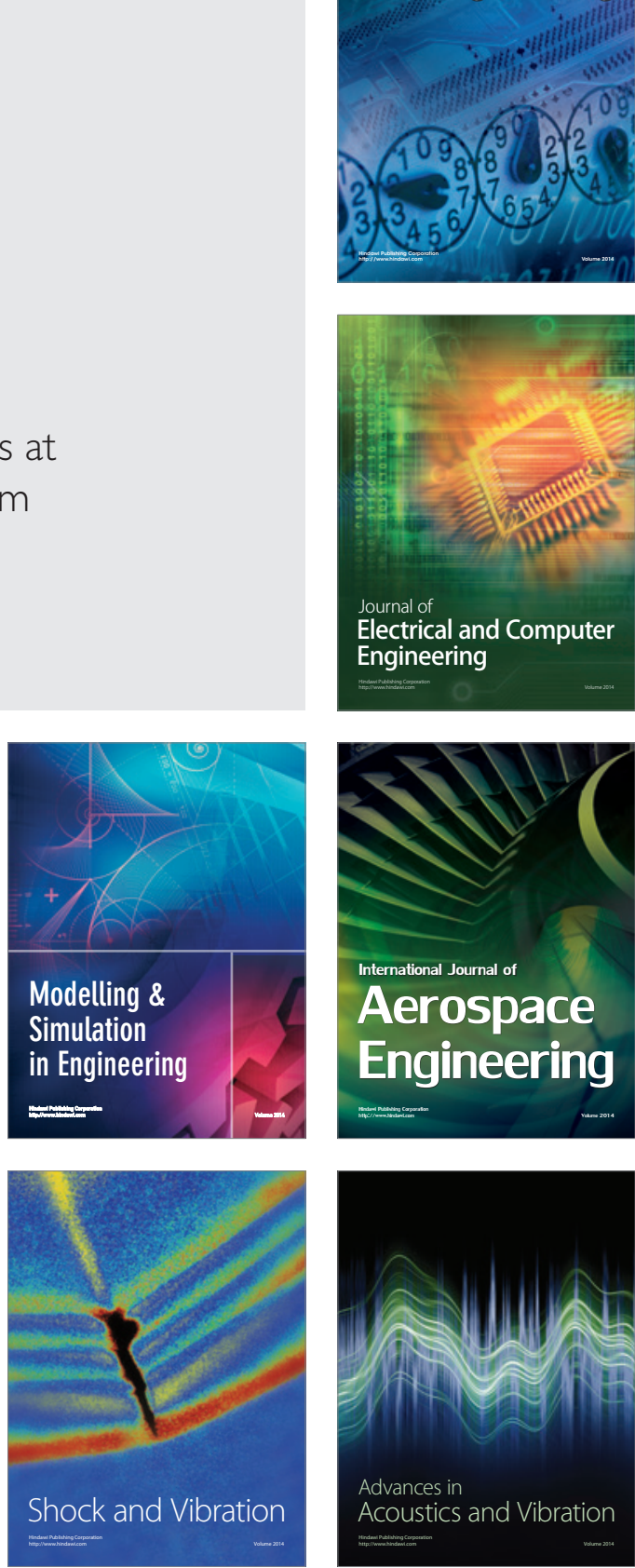